\title{
Flux emergence, flux imbalance, magnetic free energy and solar flares
}

\author{
Debi Prasad Choudhary ${ }^{\mathrm{a}, *}$, Sanjay Gosain ${ }^{\mathrm{b}}$, Nat Gopalswamy ${ }^{\mathrm{c}}$, P.K. Manoharan ${ }^{\mathrm{g}}$, \\ R. Chandra ${ }^{f}$, W. Uddin ${ }^{\mathrm{e}}$, A.K. Srivastava ${ }^{\mathrm{e}}$, S. Yashiro ${ }^{\mathrm{c}}$, N.C. Joshi ${ }^{\mathrm{e}}$, P. Kayshap ${ }^{\mathrm{e}}$, \\ V.C. Dwivedi ${ }^{g}$, K. Mahalakshmi ${ }^{\mathrm{g}}$, E. Elamathi ${ }^{\mathrm{g}}$, Max Norris ${ }^{\mathrm{a}}$, A.K. Awasthi ${ }^{\mathrm{d}}$, R. Jain ${ }^{\mathrm{d}}$ \\ ${ }^{a}$ Department of Physics \& Astronomy, California State University, Northridge, CA 91330-8268, USA \\ ${ }^{\mathrm{b}}$ National Solar Observatory, Tucson, USA \\ ${ }^{\mathrm{c}}$ NASA Goddard Space Flight Center, Greenbelt, MD 20771-0001, USA \\ ${ }^{\mathrm{d}}$ Physical Research Laboratory, Department of Space, Navrangpura, Ahmedabad 380 009, India

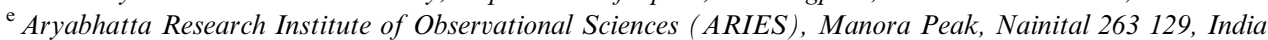 \\ ${ }^{\mathrm{f}}$ Department of Physics, DSB Campus, Kumaun University, Nainital, India \\ ${ }^{\mathrm{g}}$ Radio Astronomy Centre, NCRA, Tata Institute of Fundamental Research, Udhagamandalam (Ooty) 643 001, India
}

Received 17 January 2013; received in revised form 2 July 2013; accepted 7 July 2013

\begin{abstract}
Emergence of complex magnetic flux in the solar active regions lead to several observational effects such as a change in sunspot area and flux embalance in photospheric magnetograms. The flux emergence also results in twisted magnetic field lines that add to free energy content. The magnetic field configuration of these active regions relax to near potential-field configuration after energy release through solar flares and coronal mass ejections. In this paper, we study the relation of flare productivity of active regions with their evolution of magnetic flux emergence, flux imbalance and free energy content. We use the sunspot area and number for flux emergence study as they contain most of the concentrated magnetic flux in the active region. The magnetic flux imbalance and the free energy are estimated using the HMI/SDO magnetograms and Virial theorem method. We find that the active regions that undergo large changes in sunspot area are most flare productive. The active regions become flary when the free energy content exceeds $50 \%$ of the total energy. Although, the flary active regions show magnetic flux imbalance, it is hard to predict flare activity based on this parameter alone.
\end{abstract}

(c) 2013 COSPAR. Published by Elsevier Ltd. All rights reserved.

Keywords: Flux emergence; Flux imbalance; Magnetic free energy; Sunspot area; Solar flare

\section{Introduction}

Physical mechanisms responsible for energy storage and release in explosive events on the sun have been the subject of much serious study for many decades (Gold and Hoyle, 1959; Welsch and Longcope, 2003). In the space age, understanding of these events has practical implications (Sterling, 2000). In particular, identification of observable precursors of CMEs on the sun is an important step in space weather forecasting, as well as key to understanding the physics of magnetic explosive events.

\footnotetext{
* Corresponding author. Tel.: +1 8186777113; fax: +1 8186773234 .

E-mail address: debiprasad.choudhary@csun.edu (D.P. Choudhary).
}

The Solar flares and CMEs occur as a result of destabilization of magnetic structure above the photosphere, but their relationship is somewhat more complex as they do not often occur in the presence of each other (Andrews, 2003; Jing et al., 2006). At the same time, observations show that a major fraction, about $88 \%$ of earth directed CMEs occur following large flares (Harrison, 1968; Bemporad and Poletto, 2005; Zhou et la., 2003). The energy released from the active region magnetic field is greater prior to the CME launch than after (Green et al., 2001). Even though some CMEs are observed without a chromospheric flare, the ones originating from active regions with strong magnetic field result in higher speed ejected material (Gosling et al., 1976; MacQueen and Fisher, 
1983; Vrnak et al., 2005; Srivastava and Venkatakrishnan, 2002; ?). Clearly, there is a close relationship of flares and CMEs with the size and strength of active region magnetism as the energy released in the explosive phenomena is stored in the three dimensional magnetic structure (Gopalswamy et al., 2010). Therefore studying the precursors of solar flares is useful for CME and space weather research.

Flares happen as the active region magnetic field is destabilized. The energy is continuously added to the three-dimensional (3D) active region structure in twisted magnetic fields resulting in a stressed flux system that eventually becomes unstable (Hahn et al., 2005). As the energy builds through a series of stable non-potential configuration due to foot-point motion or emerged stressed field, the explosion is triggered by tether-cutting or break-out mechanisms due to flux emergence (Hagyard et al., 1986; Moore et al., 1992; Gary and Moore, 2004; Wang et al., 2004; Choudhary et al., 1998; Choudhary et al., 2001). Many studies relate magnetic field evolution with flares. Early work showed that the flare energy is strongly correlated with active region magnetic fields, which is reduced at least after B-class flares (Mayfield et al., 1972; Mayfield and Lawrence, 1985). Modern observations using magnetograms obtained with the GONG network showed that the field change occurs rapidly mostly at the foot-point of the flare ribbons which might result from the penumbral field relaxing upward by reconnecting magnetic fields above the surface (Sudol and Harvey, 2005; Deng et al., 2005; Wang et al., 2011). The field-guided mass motion around the neutral line also shows dramatic changes associated with flares (Deng et al., 2011). In another line of study, Falconer et al. (2009) showed that the magnetic field configuration of active regions explode after achieving their maximum attainable free energy in order to achieve the equilibrium state.

All mechanisms described above require changing the magnetic field of source regions of flares and CMEs. The sunspot area is a good measure of the localized magnetic flux which is a widely observed quantity at the locations of these source regions. Hence, along with the magnetic field measurements, using the variation in sunspot area is a powerful tool to study the temporal evolution of magnetic energy in active regions.
In this study we focus on the relation between the total energy release due to solar flares with the rate of change in sunspot area. We examine if active regions with rapidly changing sunspot area release more flare energy. Since the spot group complexity is also an indicator of flare productivity of the region, we explore the relationship with flux imbalance, which is due to twisted flux emergence among others (Green et al., 2003; Tian and Liu, 2003). Finally, we study the evolutionary stage of active region free energy content with flare occurrence.

\section{Data and analysis}

The properties of active regions such as Hale classification, sunspot area, sunspot number, flare measure in different type obtained from NOAA/USAF Active Region Summary listed in http://www.solarmonitor.org/ are given in Table 1. Fig. 1 is the histogram of active region properties during the disk passage. The active region flux emergence is measured as the time derivative of sunspot area. The total flare energy released during the disk passage is computed by adding the energy released by all flares produced by the active region,

$E_{\text {total }}=\sum_{i}\left[a * X_{i}+b * M_{i}+c * C_{i}\right]$

where the coefficients $a, b$ and $c$, represent the energy released during $X, M$ and $C$ class flares. The index $\mathrm{i}$ is the number of flares in each class.

The full disk magnetograms were obtained by the Helioseismic and Magnetic Imager (HMI) on board the Solar Dynamics Observatory (SDO). We have extracted the field of view containing the active region for successive days to compute the flux imbalance parameter and magnetic energy content. The flux imbalance is computed by,

Flux Imbalance $=\frac{B_{+}-B_{-}}{B_{+}+B_{-}}$

where $\mathrm{B}_{+}$and $\mathrm{B}_{-}$are total positive and negative magnetic field.

The total energy and free energy contained in the active region are computed using the magnetic Virial theorem (Klimchuk et al., 1992). The energy contained in a force

Table 1

Active regions.

\begin{tabular}{|c|c|c|c|c|c|}
\hline NOAA & First day of disk appearance & Complexity change & Spot area $\max / \mathrm{min}$ & Spot number $\max / \mathrm{min}$ & Flare measure \\
\hline 11283 & 2011 September 1 & $\beta \rightarrow \beta \gamma \delta$ & $230 / 100$ & $29 / 10$ & 815.8 \\
\hline 11302 & 2011 September 23 & $\beta \gamma \rightarrow \beta \gamma \delta$ & $1070 / 700$ & $26 / 10$ & 1569.6 \\
\hline 11429 & 2012 March 4 & $\beta \gamma \rightarrow \beta \gamma \delta$ & $1270 / 700$ & $28 / 8$ & 2603.6 \\
\hline 11164 & 2011 March 6 & $\beta \rightarrow \beta \gamma \delta$ & $760 / 350$ & $28 / 9$ & 129.1 \\
\hline 11158 & 2011 February 12 & $\beta \rightarrow \beta \gamma \delta$ & $620 / 40$ & $39 / 5$ & 157.4 \\
\hline 11216 & 2011 May 17 & $\alpha \rightarrow \alpha$ & $110 / 60$ & $1 / 1$ & 0 \\
\hline
\end{tabular}




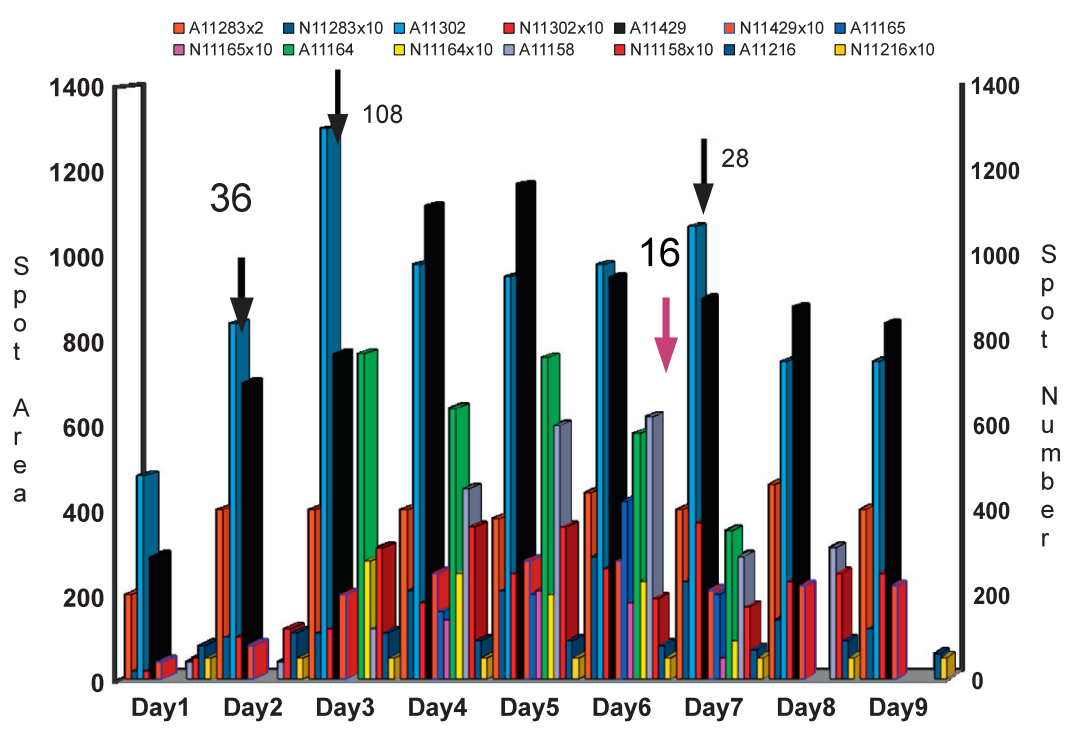

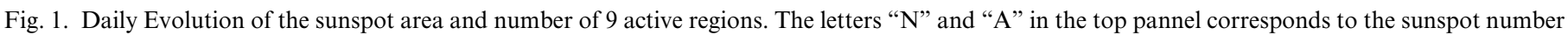
and area of the active regions. The arrow marks show the maximum number of flares occurred for a large active region.

free coronal magnetic field is given by a surface integral across the photospheric plane $(z>0)$

$E(z>0)=\frac{1}{4 \pi} \int_{z=0}\left(x B_{x}-y B_{y}\right) B_{z} d x d y$

Fractional free energy $=\frac{(\text { Total Energy }- \text { Potential Energy })}{\text { Total Energy }}$

\section{Sunspot area evolution}

Fig. 2 shows the relationship between the total flare energy released by an active region, (Flare index) as described in Section 2, with sunspot area and number. While the flare index does not show systematic dependence with either sunspot area or number in the active region, there is a clear trend with the time derivative of sunspot area. Fig. 2(a) shows the flare index as a function of the time derivative of sunspot area. During the decay phase, which is represented by the negative time derivative, the flare index is lower than the growing phase of the active region, which is consistent with an earlier finding that sudden changes in sunspot group area are associated in major flares with the pre-maximum growth (Newton and Howe, 1952). It was also found that complex spot groups produce the most flares per unit area compared to unipolar spots (Bell and Glazer, 1959). Greatrix (1963) examined nearly 4000 solar flares and found that intensity of a flare is dependent on the complexity of the geometry of the source region magnetic field and the flare intensity does not depend on the rate of change in the area of spot group. On the other hand, he found that the flare frequency is a linear function of rate of change of spot area. However, the changes in flare related sunspot area is not clear (Howard, 1963; Sivaraman, 1969). The probability of solar flare occurrence rate based on complexity and rate of change of sunspot area shows that magnetic flux emergence enhances the occurrence of major solar flares (Lee et al., 2012; Contrino et al., 2012). Recent high spatial resolution observations of sunspot configuration and magnetic field measurements show that the spot area changes due to permanent changes in magnetic field configuration (Wang et al., 2001; Liu et al., 2005). The relation of stellar flares to the spotted area on the stellar surface adds to the notion that the spot area can be used to study the flare occurrence rate and estimate the total flare energy release at the active region (Dal and Evren, 2012).

The relationship between sunspot area changes and flare occurrences has been carried out for a long time since the spot group is an approximate measure of magnetic flux passing through the active region. Thus, the relationship between solar flares and spot area can be regarded as the relation between solar flare and magnetic flux. Early observations have shown that solar flares often occur at the same locations of the active regions flux emergence (Dodson and Hedeman, 1949). We do not find the relationship of absolute value of sunspot area with flare index is consistent with the previous results.

The sunspot number dependence of the flare index shows a bimodal distribution. Regions that contain several small spots or few larger spots are flare productive as may be noticed in Fig. 2(d). However, the flare index of active regions do not show any trend. Finally, we also observed that active regions with a single sunspot that did not exhibit temporal evolution of area were without any flares.

\section{Energy estimation with magnetic Virial theorem and magneto-frictional method}

The total energy contained in the active region and its temporal evolution were used to study the flare productivity of the active regions listed in Table 1 . One part of the 

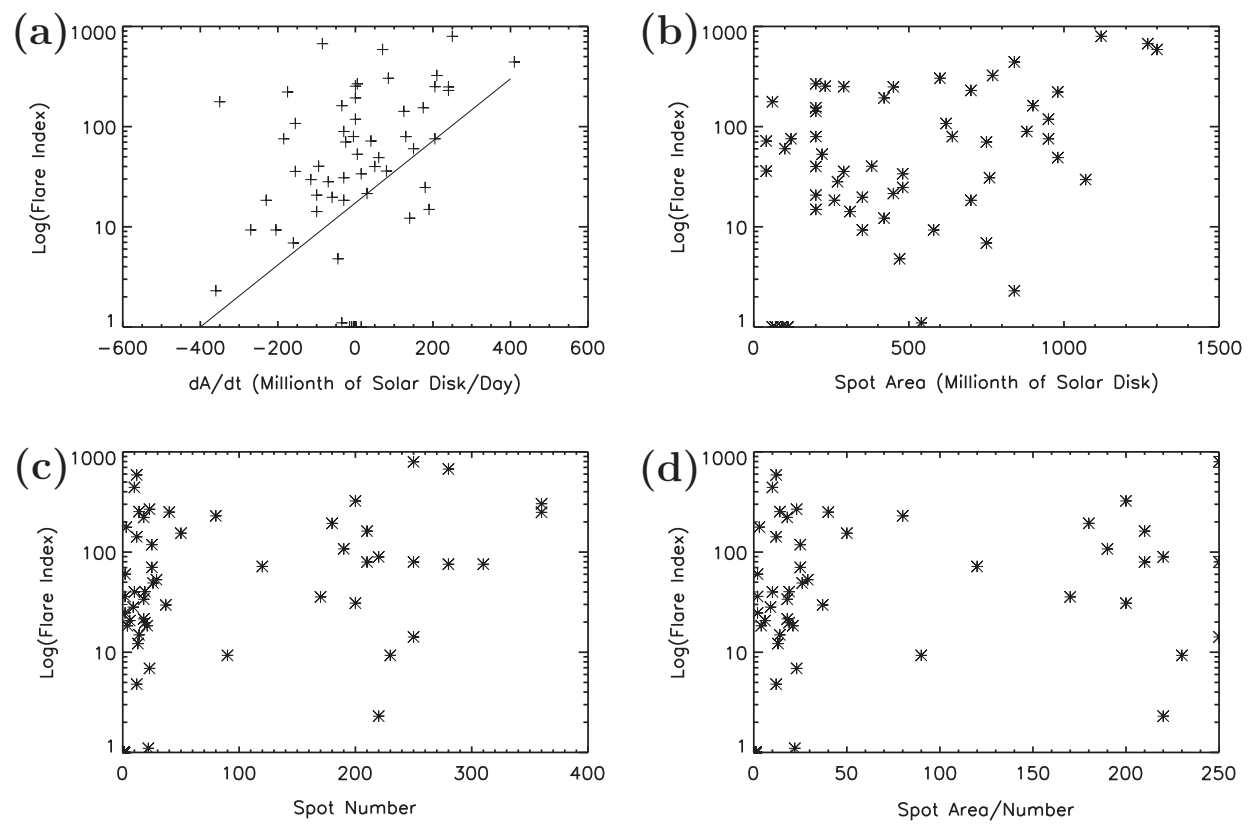

Fig. 2. Depandence of total flare index with sunspot area and number. (a) The total flare index and rate of change of sunspot area (dA/dt). The sold line is the lower limit below which most active regions do not exibit flare activity. (b) The dependent of flare index on the total spot area. (c) Dependence of flare Index on sunspot number. (d) Dependence of flare index on the sunspot to number ratio.

general Virial theorem states that, for a force free magnetic field, the energy contained within a volume is given by the surface integral over the bounding surface (Chandrasekhar, 1961, chap 13). Klimchuk et al., 1992 derived magnetic Virial theorem with reasonable assumptions and devised a useful scheme to compute the energy with observed photospheric vector magnetograms that would have noise contained in the polarization signal. We have used this method to determine the energy of active regions that will provide a basis for comparison. The Virial theorem gives the total magnetic energy, but it is only the free energy (the excess over the potential field energy) that is available to power a flare or CME. It is important to examine the free energy as a fraction of the total energy, rather than simply the total energy or the free energy. A large active region can have a lot of total energy and be potential, but it might never produce a CME or flare. Similarly, a large active region that is minimally stressed (modest deviation from potential) may have more free energy than a small active region that is highly stressed (large deviation from potential). The small active region is more likely to erupt even though it has less free energy. It is possible to compute the potential field energy using only the boundary data with the Virial theorem (Klimchuk and Sturrock, 1992). They used a magneto-frictional method to produce the desired photospheric flux distribution and field line connectivity. We implement this method to estimate the fractional free energy defined in Eq. (4), which is an indicator of an impending flare or CME. This fraction should be a rough indicator of the level of stress in the field and therefore the propensity of the field to break. Fig. 3 shows an example of the temporal evolution of estimated energy contained in magnetic field structure using magnetic Virial theorem. Two major flares occurred at the time marked with vertical dashed lines, when the magnetic free energy was high. There is also indication of decreasing free energy after the flares. Rapid time variation of energy content (may be due to flux emergence) to flares. Most of the large flares are observed at the time when the active region has free energy $>50 \%$ of total energy. Steady free energy content does not produce flares.

\section{Active region flux imbalance}

Imbalance of magnetic flux contained in the active region is another indicator of the local complex field configuration. Fig. 4 is an example of the evolution of magnetic field in terms of flux imbalance in NOAA 11283. The flux imbalance steadily increased as negative polarity continuously emerged. We find that the majority of CMEs and flares occur during or after new flux emergence that shows significant flux imbalance. The steady imbalance flux (could be due to visibility issue) does not produce flares. The flux imbalance has been observed in

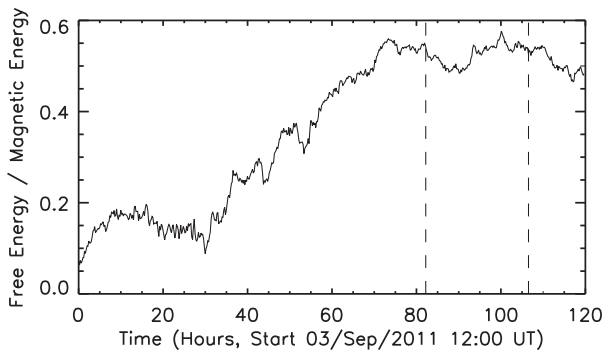

Fig. 3. Magnetic Free energy of the active region NOAA11283 as a function of time. 


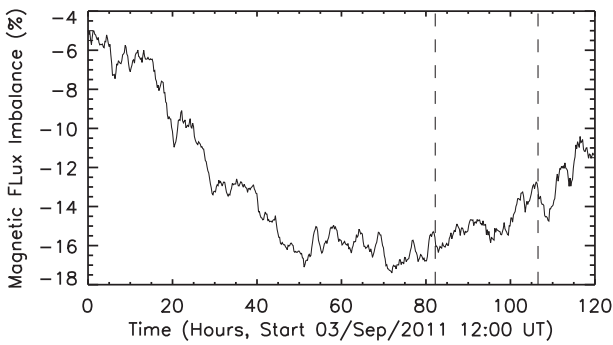

Fig. 4. Temporal evolution of the flux imbalance of active region NOAA 11283. The dashed vertical lines mark the occurrence of large solar flares.

solar active regions for a long time (see GrossmannDoerth et al., 1987). Flux imbalance can be up to $30 \%$ in ephemeral active regions and reach about 62\% (Livi et al., 1985; Sheeley, 1966; Stenflo, 1967; Choudhary et al., 2002). The local imbalance of magnetic flux in the active region can be primarily due to the emergence of tilted magnetic structure as found by analyzing more than 200 active regions (Green et al., 2003). The photospheric electric current in the field structure can produce magnetic field in several directions leading to observed imbalance of magnetic flux (Gary and Rabin, 1995). The flux imbalance can also be due to long distance connection through field lines that leads to unstable magnetic field structures (Choudhary et al., 2002). Also, the diffused nature of one of the polarity, generally the following polarity in Hale sense, can lead some flux below the detection limit that will result in a measurable flux imbalance (Grossmann-Doerth et al., 1987). Tian and Liu (2003) found that the net fluxes of seven active regions associated with strong solar storms significantly decreased within 2 or 3 days prior to the major flares associated with the storms. They also found that the flux imbalance reduced before the onsets of the flares. This suggest a possible relationship between significant decrease of the flux imbalance of active regions and instability of large-scale magnetic fields. Such phenomena can occur due to the sudden emergence of flux in preexisting magnetic field structure leading to large flares and CMEs.

\section{Conclusions}

The evolution of observed magnetic properties of solar active regions and their flare productivity is useful to develop space weather prediction tools. Several past studies exhibited partial success in relating the flare occurrence to the temporal change of magnetic field of their source region. In this paper we combine an old technique namely evolution of sunspot number and area with energy estimation with photospheric magnetic fields along with their flux imbalance. The main results of this study are:

- Evolving active regions, in which large spots (concentrated flux) emerges are flare and CME productive.

- Sudden flux emergence that results in an imbalance of photospheric flux and large flares.
- The flare activity is observed when the free energy exceeds $50 \%$ of total energy.

\section{Acknowledgment}

This research was performed as a part of the Indo-US Science and Technology Forum's Joint Center on Solar Eruptive Events.

\section{References}

Andrews, M.D. A search for CMEs associated with big flares. Solar Phys. 218, 261, 2003.

Bemporad, A., Poletto, G. A variety of coronal mass ejection: steamer puffs from compact ejective flares. Astrophys. J. 635, L189, 2005.

Chandrasekhar, S. Hydrodynamic and hydromagnetic stability. International Series of Monographs on Physics. Oxford, Clarendon, 1961.

Choudhary, D.P., Ambastha, A., Ai, G. Emerging flux and X-class flares in NOAA 6555. Solar Phys. 179, 133-140, 1998.

Choudhary, D.P., Sakurai, T., Venkatakrishnan, P. Chromospheric magnetic field of solar active regions. Astrophys. J. 560, 439-444, 2001.

Choudhary, D.P., Gosain, S., Venkatakrishna, P. On magnetic flux imbalance in solar active regions. Astrophys. J. 573, 851, 2002.

Contrino, L., Zuccarello, F., Romano, P., Spadaro, D., Guglielmino, S.L., Battiatio, V. Flare forecasting based on sunspot groups characterstics. Acta Geophys. 57, 52-63, 2012.

Dal, H.A., Evren, S. The statical analysis of flares decetcted in B band photometry of UV ceti type stars. New Astronomy 17, 399-410, 2012.

Deng, N., Liu, C., Yang, G., Wang, H., Denker, C. Rapid penumbral decay associated with an X2.3 flare in NOAA active region 9026 . Astrophys. J. 623, 1195-1201, 2005.

Deng, N., Liu, C., Choudhary, D.P., Wang, H. Rapid enhancement of sheared evershed flow along the neutral line associated with an X6.5 flare observed by Hinode. Astrophys. J. 733, L14, 2011.

Dodson, H.W., Hedeman, E.R. The frequency and position of flares within three active sunspot area. Astrophys. J. 110, 242-249, 1949.

Falconer, D.A., Moore, R.L., Gary, G.A., Adams, M. The main sequence of explosive solar active regions, discovery and interpretation. Astrophys. J. 700, L166-L170, 2009.

Gary, G.A., Moore, R.L. Eruption of a multiple-turn helical magnetic flux tube in a large flare: evidence for external and internal reconnection that fits the breakout model of solar magnetic eruptions. Astrophys. J. 611, 545-556, 2004.

Gary, G.A., Rabin, D. Sol. Phys. 157, 185, 1995.

Grossmann-Doerth, U., Pahlke, K.-D., Schssler, M. Astron. Astrophys. 176, 139, 1987.

Gold, T., Hoyle, F. On the origin of solar flares. Mon. Not. R. Astron. Soc. 120, 89-103, 1959.

Gopalswamy, N., Akiyama, S., Yashiro, S., Mkel, P. Coronal mass ejections from sunspot and non-sunspot regions, in: Hasan, S.S., Rutten, R.J. (Eds.), Magnetic Coupling between the Interior and Atmosphere of the Sun, Astrophysics and Space Science Proceedings. Springer, Berlin Heidelberg, ISBN 978-3-642-02858-8, p. 289, ISSN 1570-6591 (Print) 1570-6605 (Online), 2010.

Gosling, J.T., Hildner, E., MacQueen, R.M., Munro, R.H., Poland, A.I., Ross, C.L. The speeds of coronal mass ejection events. Solar Phys. 48, 389, 1976.

Greatrix, G.R. On the statistical relations between flare intensity and sunspots. Mon. Not. R. Astron. Soc. 126, 123-133, 1963.

Green, L.M., Demoulin, P., Mandrini, C.H., Van Driel-Gesztelyi, L. How are emerging flux, flares and CMEs related to magnetic polarity imbalance in MDI data? Solar Phys. 215, 307, 2003.

Harrison, R.A. Solar coronal mass ejection and flares. Astron. Astrophys. $162,283,1968$.

Hagyard, M.J., Rabin, D. Adv. Space Res. 6 (6), 7, 1986. 
Hahn, M., Gaard, S., Jibben, P., Canfield, R.C., Nandy, D. Spatial relationship between twist in active region magnetic fields and solar flares. Astrophys. J. 629, 1135, 2005.

Howard, R. On the relation of major solar flares with changes in sunspot area. Astrophys. J. 138, 1312-1313, 1963.

Jing, J., Song, H., Abramenko, V., Tan, C., Wang, H. The statistical relationship between the photospheric magnetic parameters and the flare productivity of active regions. Astrophys. J. 644, 1273, 2006.

Klimchuk, J.A., Sturrock, P.A. Three-dimensional force-free magnetic fields and flare energy buildup. Astrophys. J. 358, 344-353, 1992.

Klimchuk, J.A., Canfield, R.C., Rhoads, J.E. The practical application of the magnetic Virial theorem. Astrophys. J. 385, 327-343, 1992.

Livi, S.H.B., Wang, J., Martin, S.F. J. Aust. Phys. 38, 855, 1985.

Lee, K., Moon, Y.-J., Lee, J.Y., Lee, K.S., Na, H. Solar flare occurrence rate and probability in terms of the sunspot classification supplemented with sunspot area and its changes. Solar Phys., http:// dx.doi.org/10.1007/s11207-012-0091-9, 2012.

Liu, C., Deng, N., Falconer, D., Goode, P.R., Denker, C., Wang, H. Rapid changes of spot structure associated with seven major flares. Astrophys. J. 622, 722-736, 2005.

MacQueen, R.M., Fisher, R.R. The kinematics of solar inner coronal transients. Solar Phys., 89, 1983.

Mayfield, E.B., Chapman, G.A. Magnetic flux changes associated with the solar flare of August 1972. Solar Phys., 351-362, 1972.

Mayfield, E.B., Lawrence, J.K. The correlation of solar flare production with magnetic energy in active regions. Solar Phys. 96, 293, 1985.

Moore, R.L., Roumeliotis, G., in: Triggering of Eruptive Flares Destabilization of the Preflare Magnetic Field Configuration. Lecture Notes in Physics, Vol. 399. Springer-Verlag, Berlin, p. 69, 1992.
Newton, H.W., Howe, H. Area changes in sunspot and solar flare incidence. The Observatory 72, 111-117, 1952.

Sivaraman, K.R. On the relation between sunspot area changes and flare occurrences. Solar Phys. 6, 152-153, 1969.

Sheeley, N.R. ApJ 144, 723, 1966.

Srivastava, N., Venkatakrishnan, P. Relation between CME speed and geomagnetic storm intensity. Geo. Res. Lett. 29, 1287 (1 CiteID), 2002.

Sterling, A.C. Sigmoid CME source region at the sun: some recent results. J. Atom. Terr. Phys. 62, 1427, 2000.

Stenflo, J.O. Acta Univ. Lund. II, 35, 1967.

Sudol, J.J., Harvey, J.W. Longitudinal magnetic field changes accompanying solar flares. Astrophys. J. 635, 647-658, 2005.

Tian, L., Liu, Y. Tilt and alphabest of major flare-producing active regions. Astron. Astrophys. 407L, L13-L16, 2003.

Vrnak, B., Sudar, D., Ruzdjak, D. The CME flare relationship: are there really two types of CMEs? Astron. Astrophys. 435, 14, 2005.

Wang, H., Liu, C., Qiu, J., Deng, N., Goode, P.R., Denker, C. rapid penumbral decay following three X-class solar flares. Astrophys. J. Lett. 601, L195-L198, 2004.

Wang, H., Chae, J., Yurchyshyn, V., Yang, G., Steinegger, M., Goode, P. Inter-active region connection of sympathetic flaring on 2000 February 17. Astrophys. J. 559, 1171-1179, 2001.

Wang, Pu., Ding, M.-D., Ji, H.-S., Wang, H.-M. Magnetic field changes associated with three successive M-class solar flares on 2002 July 26. Res. Astron. Astrophys. 11, 692-700, 2011.

Welsch, B.T., Longcope, D.W. Magnetic helicity injection by horizontal flows in the quiet Sun. I. Mutual-helicity flux. Astrophys. J. 588, 620$629,2003$.

Zhou, G., Wang, J., Cao., Z. Correlation between halo coronal mass ejections and solar surface activity. Astron. Astrophys. 397, 105, 2003. 